\title{
Criminologie
}

\section{Les femmes et l'incarcération, le temps n'arrange rien}

\section{Louise L. Biron}

Volume 25, numéro 1, 1992

Les femmes et le contrôle pénal, questions féministes

URI : https://id.erudit.org/iderudit/017318ar

DOI : https://doi.org/10.7202/017318ar

Aller au sommaire du numéro

Éditeur(s)

Les Presses de l'Université de Montréal

ISSN

0316-0041 (imprimé)

1492-1367 (numérique)

Découvrir la revue

Citer cet article

Biron, L. L. (1992). Les femmes et l'incarcération, le temps n'arrange rien. Criminologie, 25(1), 119-134. https://doi.org/10.7202/017318ar

\section{Résumé de l'article}

Some of the issues related to the incarceration of women have long been denounced and are well documented in the current literature. This article attempts to report on the recommendations of the Task Force on federally sentenced women in Canada by indicating what were the underlying influences. Thus, the proposed policy is presented as deriving from a feminist analysis of the reality of the women in prison. It is also mentioned that, for various reasons, the implementation of such a policy may not follow the course initially intended, therefore it should be cautiously supervised.
Ce document est protégé par la loi sur le droit d'auteur. L’utilisation des services d'Érudit (y compris la reproduction) est assujettie à sa politique d'utilisation que vous pouvez consulter en ligne.

https://apropos.erudit.org/fr/usagers/politique-dutilisation/ 
Some of the issues related to the incarceration of women have long been denounced and are well documented in the current literature. This article attempts to report on the recommendations of the Task Force on federally sentenced women in Canada by indicating what were the underlying influences. Thus, the proposed policy is presented as deriving from a feminist analysis of the reality of the women in prison. It is also mentioned that, for various reasons, the implementation of such a policy may not follow the course initially intended, therefore it should be cautiously supervised.

La question des femmes et de leur incarcération donne lieu depuis quelques années à une littérature variée qui s'efforce de dénoncer à la fois les conditions d'emprisonnement et les coûts sociaux afférents (Hamelin, 1989 ; Morris, 1987; Feinman, 1986; Heidensohn, 1985, pour ne nommer que ces auteurs). De fait, l'analyse de ces problématiques a amené un certain nombre d'auteurs à réclamer des mesures de rechange à l'incarcération, celle-ci leur apparaissant tout à fait inadéquate pour répondre aux besoins spécifiques des femmes (Carlen, 1990; Dobash et al., 1986). Il est d'ailleurs symptomatique qu'on ait écrit, à cinquante ans d'intervalle, les lignes suivantes :

Vos commissaires sont d'avis qu'il est tout particulièrement important de ne pas condamner de jeunes filles à la prison.

(Ministère de la Justice, 1938, p. 151.)

Aucune femme ne devrait être incarcérée dans un pénitencier.

(Ibid., p. 154.)

Historical and contemporary evidence suggests rather that imprisonment per se is both an irrelevant and a damaging response to women's crime.

(Dobash et al., 1986, p. 214.)

1. Professeure, École đe criminologie, Université de Montréal, case postale 6128 , succ. « A », Montréal (Québec) H3C 3 J7. 
Il n'est pas possible dans ces quelques pages de reprendre tout le débat qui entoure cette question. Nous nous contenterons de présenter: 1) la clientèle et certaines conditions de détention telles qu'elles apparaissent actuellement ; 2) les approches féministes et les nouvelles orientations qu'elle sous-tendent et 3) une présentation plus détaillée des principales conclusions auxquelles sont arrivés les membres du Groupe d'étude sur les femmes purgeant une peine fédérale. L'analyse de ces conclusions devrait nous permettre de mieux cerner les orientations en matière de politique correctionnelle en ce qui concerne la clientèle féminine au Canada.

\section{CLIENTÈLE ET CONDITIONS DE DÉTENTION}

Avant de traiter des conditions de détention, il convient de brosser un portrait des femmes qui se trouvent actuellement dans des institutions carcérales.

\subsection{La clientèle}

Il existe au Canada deux types d'établissements en matière de détention, soit des institutions dites fédérales qui hébergent des femmes dont la sentence est de deux ans ou plus et des institutions dites provinciales qui regroupent des femmes qui ont une peine d'incarcération de moins de deux ans. En outre, il existe des ententes féderales-provinciales qui permettent à des détenues sous sentence fédérale de purger leur peine dans des établissements provinciaux.

Les statistiques provinciales les plus récentes, soit celles de 19881989 (Québec, 1988-1989), đénombrent 852 femmes condamnées à la Maison Tanguay à Montréal et 212 à la Maison Gomin à Québec pendant un an. Ces deux institutions sont dites provinciales. Au cours de cette même période, alors que sa capacité d'hébergement est de 132 places, la Maison Tanguay enregistre un minimum de 99 femmes et un maximum de 153. A titre indicatif, 37 femmes étaient sous sentence fédérale à la Maison Tanguay en décembre 1991, en raison d'une entente fédérale-provinciale (communication verbale).

Au Canada, en 1990, la population des femmes sous sentence fédérale est de 309 dont 69 proviennent du Québec. La très grand majorité de ces femmes sont gardées dans des institutions à sécurité maximale et 154 d'entre elles résident à la Prison des femmes, une institution dite fédérale, à Kingston. Il convient de noter qu'elles sont incarcérées le 
plus souvent pour des délits reliés aux stupéfiants et que, pour plus de 80 pour cent de ces femmes, il s'agit d'une première incarcération dans une institution fédérale. De plus, les deux tiers des femmes ont des enfants, et selon Shaw (1989), environ le quart d'entre elles ont un enfant de moins de cinq ans.

Si ces données touchent plus directement les femmes sous sentence fédérale, il convient đe noter que pour les détenues qui résident dans un établissement provincial au Québec, le profil présente les caractéristiques suivantes : pour la période de $1977-1984$, plus de 40 pour cent des femmes avaient moins de 25 ans, 61,7 pour cent étaient célibataires, 17,6 pour cent n'avaient pas atteint un niveau de scolarité secondaire et 71,9 pour cent l'avaient atteint sans nécessairement le compléter. À ce portrait s'ajoute le fait que 16,5 pour cent des femmes incarcérées avaient un emploi. En ce qui concerne les délits qui leur ont valu la détention, il s'agissait dans 60 pour cent des cas de crimes contre les biens ou d'infractions à la circulation routière. Enfin, les deux tiers (34,2\%) de cette population ont moins de trente ans (Cousineau et al, 1986). À quelles conditions de détention est soumise cette clientèle ?

\subsection{Conditions de détention}

Rendre compte de la spécificité des conditions de détention de la clientèle féminine et prendre en considération leurs besoins bien particuliers dépasse largement le cadre de cet article à moins de nous restreindre à une simple nomenclature. En conséquence, nous n'avons retenu ici que les éléments sur lesquels les analyses sont généralement tombées d'accord. La question des besoins, pour sa part, sera intégrée dans la section consacrée aux travaux du Groupe d'étude. La récurrence des problèmes liés à la sécurité, à l'éloignement et aux programmes justifie donc une attention particulière et nous autorise à les aborder plus en détail.

\subsubsection{Sécurité}

Que les établissements de détention pour femmes soient sur-sécuritaires n'apparaît pas comme un fait nouveau. Il y a plus de vingt ans, le rapport Ouimet (Solliciteur général du Canada, 1969) en faisait état dans son chapitre consacré à la femme contrevenante.

L'architecture carcérale de même que le peu d'institutions pour femmes, en général, font en sorte qu'un seul genre, extrêmement contraignant, sert de modèle et impose un niveau de sécurité maximale à toute la population féminine. Compte tenu de la nature et du volume 
des délits commis par les femmes, en général, il est pour le moins étonnant de constater que la conception des lieux physiques reflète une tout autre réalité. Cet état de fait est d'ailleurs relevé par Morris (1987, p. 122) en Angleterre, par Feinman (1989, p. 49) aux États-Unis et par Berzins et Collette-Carrière (1979, p. 93) et Elliot et Morris (1987, p. 150) au Canada. La situation ne semble guère s'améliorer si l'on en juge par les propos de Feinman qui révèle que, suite à des pressions du public, lui-même influencé par les images que renvoient les médias, on assiste à l'érection de clôtures hérissées de lames de rasoir autour de plusieurs institutions carcérales pour femmes aux États-Unis (p. 48).

Déjà en 1983, Pat Carlen soulignait le caractère paradoxal des conditions de sécurité appliquées aux femmes en détention. Elle observait, en effet, que l'administration impose des règles très strictes à des femmes qui reviennent constamment dans le système, que toute l'administration connaît et qui ont de très courtes sentences en raison de délits mineurs (p. 91). Par ailleurs, les plus longues sentences pour des délits plus graves s'administrent souvent de façon plus souple en prévoyant des programmes de sortie pour la formation scolaire ou professionnelle. Carlen (1983) en conclut que les prisons sont conçues de par leur structure et leur réglementation pour répondre à des besoins de masse et qu'elles souffrent difficilement les cas d'exception.

Il est également intéressant de noter, comme le font d'ailleurs Dobash et al. (1986), que dans la conception des prisons, il est très peu question de politique en matière de règlements et de punitions mais que dans la vie de tous les jours ces aspects ont une importance très grande à la fois pour le personnel et les détenues (p. 147).

Il s'ensuit qu'un grand nombre de règles, certaines plus triviales que d'autres, régissent la vie quotidienne des femmes incarcérées et que la désobéissance à l'une ou l'autre de ces règles enclenche tout un système de pertes de privilèges ou d'argent. D'aucuns considèrent également qu'il semble y avoir des règlements plus sévères pour les femmes que pour les hommes (Morris, 1987; Dobash et al., 1986). Parent (1991) résume bien la situation quand elle affirme que "le contrôle pénal n'est qu'une facette du contrôle exercé sur les femmes, qu'une instance où se reproduisent leur conditions d'oppression" (p. 246).

Si les conditions en matière de sécurité ne conviennent pas aux besoins et à la réalité des femmes incarcérées, qu'en est-il de la question de l'éloignement? 


\subsection{2 Éloignement}

Que les femmes soient détenues dans des établissements qui sont situés loin de leur lieu de résidence constitue un fait. Ainsi, au Canada, jusqu'au moment des ententes fédérales-provinciales des années 1970, la seule institution carcérale pour femmes sous sentence fédérale était la Prison des femmes à Kingston, en Ontario.

Cette situation n'est certes pas propre au système pénal canadien. Feinman (1986) la déplore en indiquant le paradoxe auquel donnent lieu une criminalité commise majoritairement dans un contexte urbain et la localisation des centres de détention en milieu rural. Aux États-Unis, où les minorités sont surreprésentées dans les populations carcérales, Feinman note que l'éloignement constitue un problème réel. Le même phénomène s'observe au Canada, en particulier pour les autochtones.

Le fait qu'il y ait peu de femmes en détention a longtemps servi de prétexte à la centralisation des maisons de détention et à l'érection de bâtiments qui abriteraient l'ensemble de la population carcérale féminine. Ceci a donné lieu à des déracinements majeurs en raison même des vastes territoires couverts.

Les conséquences de cet éloignement sont nombreuses. Qu'il suffise de mentionner la rupture des liens affectifs et les difficultés de la réinsertion sociale. De fait. d'aucuns soulignent l'importance de la relation affective pour les femmes en détention (Giallombardo, 1966 ; Dobash et al., 1986; Hamelin, 1989). Or, l'éloignement entraîne des difficultés et dans certains cas une impossibilité de maintenir des liens avec la famille ou les amis parce que les visites sont trop onéreuses. Dans le cas des femmes qui ont des enfants, il devient à peu près impensable d'entretenir une relation suivie. Au pire, les enfants sont confiés à une agence sociale, au mieux ils sont confiés à un membre de la famille mais. dans tous les cas, la rupture est dramatique.

En ce qui concerne la réinsertion dans leur milieu, la situation n'est guère plus facile. Comment préparer à la sortie une femme qui habite à des centaines ou des milliers de kilomètres de l'institution carcérale et qui, de surcroît, appartient à une culture différente de celle qui prévaut dans le milieu de détention? La solution ne peut résider uniquement dans l'attribution de conseillers qui assurent une liaison entre l'institution et le milieu. Cette mesure constitue, au mieux, un pis-aller.

Les conditions de sécurité et l'éloignement contribuent chacun à leur façon à une surenchère des coûts absorbés par les femmes en détention. Qu'en est-il des programmes ? 


\subsubsection{Programmes}

Une étude fort complète entreprise par Glick et Neto aux ÉtatsUnis (rapportée par Feinman, 1986) auprès de 6466 femmes détenues révèle que moins de 60 pour cent de ces femmes ont complété une scolarité équivalente au secondaire et que 15 pour cent n'ont atteint qu'un niveau élémentaire. Bien qu'aucune étude de cette envergure n'ait été effectuée au Canada, il y a lieu de croire que les résultats seraient sensiblement les mêmes ici.

Nous avons noté précédemment que peu de femmes ont un emploi ou une formation spécifique qui leur garantisse un accès plus facile au marché du travail. Dobash et al. (1986) résument bien la question des programmes en institution quand ils affirment que les auteurs qui se sont penchés sur la question des femmes en prison ont accordé peu d'importance à des aspects tels le travail, la formation professionnelle ou le perfectionnement scolaire.

En effet, l'accent a été mis sur le traitement et sur la réhabilitation, celle-ci visant surtout à rétablir la femme dans son rôle social de mère, d'épouse et de gardienne du foyer et à la faire adhérer aux valeurs de la classe moyenne. Et même si Morris (1987) considère que les changements sont plus apparents dans les institutions pour femmes aux ÉtatsUnis qu'en Angleterre, il s'agit de gains mineurs et encore loin d'être généralisés.

En fait, la remarque de Giallombardo prononcée en 1966 reste actuelle : "An attempt on the part of an inmate to further her own interests in the prison situation clearly conflicts with official policy, as the structure of the situation specifies that the inmate subordinate her own interests to those of the larger collectivity" (p. 64).

Dans un contexte où la majorité des femmes incarcérées n'ont jamais connu une trajectoire de travail relativement stable, où les emplois occupés les rejettent habituellement dans la catégorie des travailleurs non-spécialisés donc mal rémunérés (Feinman, 1986) et où la société exige une main-d'œuvre de plus en plus formée, ne faut-il pas s'étonner de constater que les programmes soient surtout confinés à des tâches traditionnellement réservées aux femmes (cuisine, buanderie, couture, coiffure)?

En 1977, Smart faisait deux remarques importantes. D'une part, elle notait : 
Most regimes employed in penal institutions for female offenders are typically those which reinforce the stereotypical, traditional sex role of women in our culture (p. 140).

Et d'autre part, elle portait le jugement suivant :

It would seem from this statement that policy-makers believe that women in prison are not worth, or capable of, retraining (p. 141).

Parallèlement, l'enquête de Shaw (1989) révèle que la situation des femmes au Canada s'apparente à celle qui vient d'être décrite et que les femmes rencontrées réclament une meilleure préparation au marché du travail actuel. À ces considérations, s'ajoute la dimension linguistique qui s'avère certainement d'une très grande importance pour les femmes francophones incarcérées dans une institution anglophone.

Ainsi, il n'a pas toujours été possible pour les femmes d'assumer pleinement leurs responsabilités sur le plan économique, pas plus qu'il ne leur a été facile d'intégrer le marché du travail. Ceci tient en partie à l'état de dépendance dans lequel elles ont souvent été maintenues. En conséquence, il semble difficile de concevoir des programmes sans que ces mêmes programmes s'inscrivent dans une perspective plus générale de développement d'habiletés personnelles. Cette vision globale constitue l'une des pierres angulaires de l'approche féministe, et il sera possible d'en mesurer l'impact sur les politiques pénales pour les femmes dans les sections suivantes.

\section{LES FÉMINISTES ET LA QUESTION PÉNALE}

Depuis les années 1970, une abondante littérature a révélé plusieurs aspects de la discrimination qui s'exerce sur les femmes dans le contexte de la justice pénale. La dénonciation porte à la fois sur les questions législatives (Boyle et al., 1985 ; Smart, 1989) et leurs applications (Chesney-Lind, 1973; Moyer et White, 1981; Zingraff et Thompson, 1984). En outre, il est intéressant de constater que les travaux axés sur la comparaison des hommes et des femmes ont lentement fait la place à un questionnement sur la place même de la femme dans le système pénal (Currie, 1986), Car comme le dit Cain (1990), les travaux sur l'égalité ou la non-égalité du traitement ne risquent-ils pas de faire oublier des questions de justice plus fondamentales? Pour reprendre les termes de Parent (1991), "il est sans doute plus fructueux de considérer le traitement pénal comme différentiel selon le sexe » (p. 297). 
Dans ce contexte, il s'agit moins d'opérer des comparaisons quantitatives et de tenter de mesurer des degrés de différence que de mesurer l'impact du système pénal selon qu'il est vécu par l'homme ou par la femme. Il s'ensuit que des méthodes qui permettent d'évaluer qualitativement ces différences apparaissent plus appropriées que des comparaisons de nombres, surtout quand les populations sont à ce point disproportionnées, comme c'est le cas dans le système pénal. À ceci, Simpson (1989) ajoute que l'accent placé par les féministes sur des méthodologies plus souples ne peut que profiter à la criminologie.

Voici donc le cadre dans lequel s'articule une réflexion sur le plan épistémologique. Si cette remise en question semble plus visible depuis quelques années, elle a néanmoins été amorcée au fil des ans par Heidenshon (1968), Klein (1973), Smart (1977), Bertrand (1979), Carlen (1988), Daly et Chesney-Lind (1988) entre autres. Bien qu'il ne soit pas possible dans ces quelques lignes de reprendre l'apport spécifique de ces auteurs, il convient certainement de rappeler la pertinence d'une pensée féministe en criminologie telle que formulée par Daly et Chesney-Lind dans leur synthèse en 1988. Elles affirment que les féministes ont contribué à l'étude du crime et de la justice pénale de quatre façons que nous résumons ici.

Premièrement, les féministes ont intégré la notion de sexe dans leur cadre conceptuel, ce qui a pour conséquence de raffiner et d'enrichir leur paradigme. Deuxièmement, les féministes ont décloisonné la discipline en puisant des concepts et des paramètres dans d'autres disciplines, qu'on pense à l'histoire par exemple. La criminologie a donc bénéficié d'un regard différent, d'un apport qui permet la remise en question d'un certain nombre de postulats. Troisièmement, elles ont permis de nous éloigner d'un modèle d'analyse conçu pour l'homme blanc moyen, montrant que ce modèle ne peut s'appliquer d'une façon générale à tous et que la question des femmes, en particulier, peut difficilement être étudiée dans le cadre de ce modèle unique. Finalement, les études féministes ont permis la congruence de plusieurs courants théoriques différents parce qu'elles transgressent des limites établies arbitrairement. Daly et Chesney-Lind en concluent que c'est une telle congruence qui permettra une articulation théorique capable d'intégrer les rapports entre classe, race et sexe en criminologie.

La criminologie dont il est question ici est centrée sur les femmes. Leur vie, leurs expériences en constituent les points de départ. Cette approche a un double mérite. D'abord, elle évite un piège contre lequel nous met en garde Parent (1991) quand elle craint que les analyses qui 
contribuent à renouveler des enjeux paradigmatiques puissent desservir les femmes en éloignant la pensée féministe de la discipline criminologique (p. 338); bien au contraire, on assiste ici à une alliance. Ensuite, elle nous rapproche des fondements de l'intervention féministe (Corbeil et al., 1983). En effet, celle-ci propose de s'attaquer aux dimensions sociétales des rôles des femmes en plus de porter attention aux dimensions personnelles. Elle s'appuie également sur le fait que les femmes doivent, au cours d'un processus d'aide, assumer leur responsabilité personnelle. Le fait de considérer d'une façon globale les questions qui touchent les femmes, leur intégration de même que l'identification de plusieurs points d'ancrage de leurs problèmes dans un contexte social plus vaste qui rejoigne toutes les femmes nous entraîne bien loin du modèle traditionnel d'intervention auquel nous avons été habitués. Nous verrons, dans les lignes qui suivent, comment ces diverses notions ont inspiré la politique actuelle au Service correctionnel du Canada.

\section{UNE APPROCHE FÉMINISTE APPLIQUEEE AUX FEMMES SOUS SENTENCE FÉDÉRALE}

Après plusieurs années de discussions, de recommandations provenant de divers groupes et commissions et de contestations qui viennent de la clientèle et des organismes communautaires, le Service correctionnel du Canada formait en 1989 un groupe d'étude qui devait se pencher, une fois de plus, sur la question des femmes sous sentence fédérale. Les membres de ce groupe représentent les services correctionnels, les organismes communautaires et ceux qui sont voués à la cause des femmes ${ }^{2}$. Il s'agit même d'une expérience unique, puisque ce groupe d'étude est co-présidé par un représentant du Service correctionnel et par une représentante d'un organisme communautaire.

Avant même de commencer ses travaux, le groupe d'étude a énuméré un certain nombre de principes qui découlent directement des réflexions féministes dont nous faisions part précédemment.

Ainsi, le groupe d'étude s'est d'abord prononcé contre le recours à l'incarcération, considérant que la nature et le volume de la délinquance des femmes ne peut en aucune façon justifier cette peine extrême. Ensuite, il a été convenu de ne jamais comparer la situation des femmes à celle des hommes en détention ; l'homme n'est donc plus le point de

2. Il convient de dire que l'auteure faisait partie de ce groupe à titre de représentante communautaire du Québec. 
référence. Il s'ensuit que les besoins des femmes constituent le point de départ dans la conception des services et des programmes destinés aux femmes. Finalement, l'approche privilégiée suppose une vision globale de la femme et de ses besoins, vision qui dépasse largement le pénal.

Compte tenu de ces principes généraux, qu'en est-il des recommandations du groupe d'étude et comment s'oriente la politique pénale du service correctionnel vis-à-vis sa clientèle féminine? Elle s'articule autour de l'énoncé de principe suivant:

Le Service Correctionnel du Canada avec le soutien des communautés a la responsabilité de créer un environnement qui habilite les femmes purgeant une peine fédérale à faire des choix responsables et valables leur permettant de vivre dans la dignité et le respect.

(Service correctionnel du Canada, 1990, p. 143.)

De cet énoncé découlent un certain nombre de recommandations. La première porte sur le logement.

\subsection{Le logement}

L'institution actuelle, la Prison des femmes, doit être fermée et remplacée par cinq établissements régionaux situés à Halifax, Montréal, au sud-ouest ou au centre de l'Ontario, à Edmonton et dans le sud-ouest de la Colombie-Britannique. En outre, une loge de guérison est prévue pour les autochtones; son architecture de même que son aménagement relèvent des directives de la communauté autochtone.

Toute une série de lignes directrices touchant l'aménagement du paysage, la taille des maisons, leur nombre, leur fonction, etc., sont prévues dans le rapport du groupe d'étude. Ces propositions, bien qu'elles ne s'en inspirent pas directement, s'apparentent à celles qui ont orienté les changements à l'institution de Shakopee au Minnesota.

Ces considérations visent à créer un environnement flexible et sain qui s'apparente autant que possible à la vie en société. Il s'agit d'une stratégie qui permet, le plus rapidement possible, la réintégration des femmes dans leur milieu. Parallèlement, le groupe d'étude préconise des centres communautaires qui peuvent être des maisons de transition, des foyers privés ou encore des centres de traitement pour toxicomanie, par exemple, et qui facilitent le rapprochement des femmes avec leurs proches. Il convient de noter que l'aspect sécuritaire n'est nullement invoqué. En effet, le groupe d'étude a été saisi du problème de la sécurité et de l'abus qu'on en a fait. Ainsi, il est intéressant de noter que 
lors de l'entente avec la Maison Tanguay, en 1982, rien n'a été prévu au chapitre des programmes alors qu' on traitait du problème de la sécurité. On dit même qu' «environ un million de dollars a été consacré à la rénovation de l'établissement pour le rendre plus sécuritaire " (Service correctionnel du Canada, 1990, p. 82).

Le souci d'utiliser de façon constructive les besoins et les ressources des femmes nous éloigne également d'une évaluation centrée sur des niveaux de sécurité. Il n'est jamais question de sécurité minimum ou maximum, ces termes relevant d'un modèle masculin. Les recommandations font état de la nécessité de responsabiliser la femme quant à son destin et aux moyens qu'elle compte prendre pour se donner des choix véritables. Dans l'esprit du groupe d'étude, le programme architectural doit être associé à une conception novatrice des divers programmes qui seront offerts et à un aménagement des lieux qui facilitent le contact de la mère avec ses enfants.

\subsection{Les programmes}

Les programmes occupent bien évidemment une place importante dans tous les établissements. Ceux-ci doivent également respecter deux principes: 1) répondre aux besoins et aux désirs exprimés par les femmes ; 2) provenir des services offerts par la communauté. Dans les deux cas, il s'agit d'appuyer la femme dans sa démarche d'autonomie et d'assurer son intégration dans un milieu ouvert de telle sorte qu'elle puisse poursuivre sa démarche, à la fin de sa sentence, en comptant sur les ressources communautaires.

Si certains des programmes tombent dans la catégorie des service de santé, de counselling ou de toxicomanie, services qu'on pourrait qualifier d'essentiels, d'autres, non moins importants, touchent les études et la formation professionnelle. Le groupe d'étude met l'accent sur l'amélioration des compétences et ses recommandations suggèrent d'individualiser la formation et les services offerts de manière à habiliter la femme à s'intégrer au marché du travail selon ses compétences.

À ces formations polyvalentes et à ces divers programmes s'ajoutent des recommandations sur l'intégration des bénévoles dans la vie de tous les jours et l'importance du loisir, de l'exercice physique, du plein air, des activités de groupe. Le groupe d'étude ne s'est pas prononcé clairement sur la question de l'intégration des enfants en milieu carcéral. Par ailleurs, afin de pallier les difficultés de maintenir des liens avec les enfants, il a recommandé : 1) des aménagements qui facilitent 
les contacts et 2) une assistance dans la mesure où les mères peuvent la requérir.

Tous ces éléments sont également repris avec une orientation particulière quand il s'agit de la clientèle autochtone. Outre une loge de guérison qui constitue un apport significatif, on prévoit des aménagements et des programmes conçus par les autochtones. Il est entendu que ces recommandations constituent une étape intérimaire puisque, idéalement, il devrait y avoir un système judiciaire autochtone (p. 100).

Bien que les recommandations touchant les aménagements physiques, le niveau de sécurité, le support ou la thérapie, la formation et la réinsertion sociale visent à innover, elles n'en demeurent pas moins ancrées dans un contexte de détention. Et voilà le paradoxe auquel se heurte le groupe d'étude. En effet, on y lit :

Le Groupe d'étude croit que la société doit s'engager sur la voie qui mènera, à long terme, à l'adoption de mesures de justice réparatrice qui aient un caractère communautaire.

(Service correctionnel du Canada, 1990, p. 100.)

Il s'agit d'une volonté politique, dans la mesure où il n'est question, pour le moment, que d'intentions et de principes, il convient donc d'être extrêmement vigilant pour que l'implantation de ces recommandations ne s'écarte pas résolument du projet initialement prévu, en particulier en ce qui touche l'aspect communautaire. Les impératifs bureaucratiques, les ressources sur place, le personnel de même que les investissements immobiliers constituent autant de facteurs qui pourraient compromettre la réalisation des objectifs énoncés.

En outre, malgré l'intérêt indéniable des recommandations du groupe d'étude. elles ne s'écartent pas d'un idéologie de réforme, réforme amorcée par Elizabeth Fry et Mary Carpenter en Angleterre, à la fin du siècle dernier. En fait, ces pionnières reconnaissaient déjà que les besoins des femmes incarcérées pouvaient être différents de ceux des hommes, mais elles ne suggéraient que des moyens pour améliorer les conditions de détention des femmes sans remettre en question le principe de la détention.

\section{CONCLUSION}

Les études féministes font depuis quelques années des gains importants sur le plan épistémologique dans la mesure où elles facilitent la 
redéfinition des concepts et contribuent à l'enrichissement des paradigmes en criminologie. Elles s'appuient également sur des approches méthodologiques non traditionnelles pour analyser leurs hypothèses.

Les analyses féministes s'exposent toutefois à stagner dans le domaine pénal si elles se confinent à des stratégies de changement qui ne visent qu'à améliorer les conditions actuelles des femmes. Le dilemme est de taille : comment réconcilier les concepts et les principes d'une part, avec l'application des politiques pénales, d'autre part. En effet, des femmes détenues vivent dans des régimes de vie qui semblent calqués sur une vision du passé, comme il a été possible de le constater dans les pages précédentes. Dans un tel contexte, comment ne pas chercher à remédier à cette situation et ce, dans les plus brefs délais. $\mathrm{Ce}$ fut l'orientation qu'a prise le groupe d'étude, et bien qu'il soit encore trop tôt pour en apprécier les mérites, il existe, à tout le moins, une volonté politique très claire pour en assurer le suivi.

Par ailleurs, il convient d'amorcer une réflexion en profondeur qui ébranle les fondements mêmes du système pénal, réflexion qui peut difficilement avoir des retombées à court terme et qui entraîne un débat d'une grande complexité chez les féministes. Cela pose un autre défi, ainsi que le notait Parent (1991):

En limitant le pouvoir du pénal et en cherchant des solutions aux problèmes des femmes ailleurs que dans le droit, nous pourrons peut-être échapper aux limites connues jusqu' ici sans renoncer à venir en aide aux femmes (p. 286).

Ainsi, dans la mesure où l'influence des féministes joue dans la définition des questions pénales et la façon de les traiter, on peut espérer que cette influence va également se faire sentir dans la résolution des conflits et dans la réparation du tort causé et que les solutions apportées appartiendront à un univers beaucoup plus large que celui auquel nous avons été confinés. La conjoncture semble favorable à de véritables changements ; pouvons-nous enfin espérer ne pas devoir reprendre cette même réflexion dans quelques années? 


\section{BIBLIOGRAPHIE}

BERTRAND, Marie-Andrée (1979), La Femme et le crime, Montréal, L'Aurore.

BERZINS, L. et Collette-Carrière, R. (1979), « La femme en prison. Un inconvénient social! », Santé mentale au Québec, vol. IV, $\mathrm{n}^{\circ} 2$, novembre.

BOYLE, C., BERTRAND, M.-A., LACERTE-LAMONTAGNE, C. et SHEMAI, R. (1985), « Un examen féministe du droit criminel », Condition féminine Canada.

CAIN, M. (1990), « Towards Transgression : New Directions in Feminist Criminology ", International Journal of the Sociology of Law, vol. 18, $\mathrm{n}^{\circ}$ 1, pp. 1-18.

CANADA (1990), Rapport du profil de la population féminine. Service des renseignements de la gestion, Service correctionnel du Canada, 12/31/90.

CARLEN. Pat (1983), Women's Imprisonment. A Study in Social Control, Boston, Routledge \& Kegan Paul.

CARLEN, Pat (1988), Women, Crime and Poverty, Milton Keynes, Open University Press.

CARLEN, Pat (1990), Altematives to Women's Imprisonment, Milton Keynes, Open University Press.

CHESNEY-LIND, Meda (1973), « Judicial Enforcement of Female Sex Role: The Family Court and the Female Delinquent $»$, Issues in Criminology, vol. 8, $\mathrm{n}^{\circ} 2$, pp. 51-69.

CHESNEY-LIND, Meda (1978), "Chivalry Reexamined: Women and the Criminal Justice System », in L. H. Bowker (ed.), Women, Crime and the Criminal Justice System, Lexington, Mass., Lexington Books, pp. 197-223.

CORBEL, C., PÂQUET-DEEHY, A., LAZURE, C. et LEGAULT, G. (1983), L'Intervention féministe, Montréal, Éditions coopératives Albert Saint-Martin.

COUSINEAU, M. M., LABERGE, D. et THEORET, B. (1986), Une analyse de la détention provinciale québécoise durant la dernière décennie, rapport de recherche, Montréal, Université du Québec à Montréal.

COUSINEAU, M. M., GARCEAU, M.-L., LABERGE, D., NERON, J. et THÉORET, B. (1988), Mémoire présenté au comité permanent de la justice et du Solliciteur général de la Chambre des communes du Canada, Les Cahiers du GRAAAP, Centre intemational de criminologie comparée, Université de Montréal, département de sociologie, Université du Québec à Montréal.

CURRIE, Dawn (1986), «Female Criminality : A Crisis in Feminist theory, in BRIAN D. MacLEAN (éd.), The Political Economy of Crime, Alger Press, chap. 14.

DALY, K. et CHESNEY-LIND, M. (1988), « Feminism and Criminology», Justice Quarterly, vol. 5, $\mathrm{n}^{\circ} 4$, pp. 497-538.

DOBASH, RUSSELL P., DOBASH, R., EMERSON et GUTTERIDGE, Sue (1986), The Imprisonment of Women, Oxford, Basil Blackwell. 
ELLIOT, Liz, MORRIS, Ruth (1987), "Behind Prison Doors *, in Ellen Adelberg et Claudia Currie (éd.) Too Few to Count, Vancouver, Press Gang Publ., pp. 145162.

FEINMAN, Clarice (1986), Women in the Criminal Justice System, $2^{c}$ edition, Toronto, Praeger Publ.

GIALLOMBARDO, R. (1966), Society of Women : A Study of Women's Prison, Toronto, John Wiley \& Sons.

HAMELIN, Monique (1989), Femmes et prison, Montreal, Éditions du Méridien.

HEIDENSOHN, Frances (1968), « The Deviance of Women : A Critique and an Enquity ", British Journal of Sociology, vol. 19, $\mathrm{n}^{\circ} 2$, pp. 160-176.

HEIDENSOHN, Frances (1985), Women \& Crimes, New York, New York University Press.

HEIDENSOHN, Frances (1986), «Models of Justice : Protia or Persephone? Some Thoughts on Equality, Faimess and Gender in the Field of Criminal Justice ", International Journal of Sociology of Law, vol. 14, pp. 287-298.

JUSTICE CANADA (1938), Commission royale d'enquête sur le système pénal du Canada, Ministère de la Justice.

KLEIN, D. (1973), "The Etiology of Female Crime : A View of the Literature », Issues in Criminology, vol. 8, $\mathrm{n}^{\circ} 2$, pp. 3-30.

KLEIN, D. et KRESS, J. (1976), "Any Woman's Blues : A Critical Overview of Women, Crime and the Criminal Justice System » Crime and Social Justice, vol. 5 , pp. 34-47.

MORRIS, Allison (1987), Women, Crime and Criminal Justice, Oxford, Basil Blackwell Ltd.

MOYER, I. L., WHITE, G. F. (1981), « Police, Processing of Female Offenders », in L. H. Bowker (éd.), Women and Crime in America, New York, MacMillan, pp. 366-377.

PARENT, Colette (1991), Les Féminismes et la question paradigmatique en criminologie, Université de Montréal, École de criminologie, thèse de doctorat.

QUÉBEC (1988-1989), Statistiques correctionnelles, Direction générale des services correctionnels, Ministère de la sécurité publique.

SERVICE CORRECTIONNEL DU CANADA (1990), La Création de choix, rapport du Groupe d'étude sur les femmes purgeant une peine fédérale.

SHAW, M. (1989), Sondage auprès des fenmes purgeant une peine fédérale, rapport intérimaire, Solliciteur genéral, Canada, Ottawa.

SIMPSON, Sally, S. (1989), "Feminist Theory, Crime and Justice ", Criminology, vol. $27, n^{\circ} 4$, pp. 605-631.

SMART, C. (1977), Women. Crime and Criminology. A Feminist Critique, New York, Routledge \& Kegan Paul. 
SMART, C. (1989), Feminist and the Power of Law, New York, Routledge.

SOLLICITEUR GÉNÉRAL DU CANADA (1969), Comité canadien de la réforme pénale et correctionnelle, Ministère du Solliciteur général du Canada.

ZINGRAFF, M., THOMPSON, R. (1984). « Differential Sentencing of Women and Men in the USA $»$, International Journal of the Sociology of Law, vol. 12, pp. 401-413. 